\title{
ARTICLE \\ Diazepam limits microglia-mediated neuronal remodeling in the prefrontal cortex and associated behavioral consequences following chronic unpredictable stress
}

\author{
Justin L. Bollinger ${ }^{1}$, Matthew J. Horchar ${ }^{1}$ and Eric S. Wohleb ${ }^{1}$
}

\begin{abstract}
Chronic stress induces neuronal atrophy and synaptic loss in the medial prefrontal cortex (PFC), and this leads to behavioral and cognitive impairments. Our recent findings indicate that microglia contribute to structural remodeling of neurons via increased colony-stimulating factor (CSF)-1 in the medial PFC. Other work shows that chronic stress induces aberrant neuronal activity in the medial PFC, and that neuronal hyperactivity increases CSF1 signaling and alters microglia function. Thus, the present studies were designed to examine the role of neuronal activity in stress-induced CSF1 signaling and microglia-mediated neuronal remodeling in the medial PFC. Additional analyses probed stress effects on the dorsal hippocampus (HPC), basolateral amygdala (BLA), and somatosensory cortex (SSCTX). Mice were exposed to chronic unpredictable stress (CUS) or handled intermittently as controls, and received daily injection of vehicle or diazepam (1 mg/kg). As anticipated, diazepam attenuated CUS-induced behavioral despair and cognitive impairments. Further studies showed that diazepam normalized Csf1 and C3 mRNA in the PFC, and prevented increases in Csf1r and $C d 11 b$ in frontal cortex microglia following CUS. Stress had no effect on neuroimmune gene expression in the HPC. Confocal imaging in Thy1-GFP(M) mice demonstrated that diazepam limited microglial engulfment of neuronal elements and blocked CUS-induced dendritic spine loss in the medial PFC. Altogether, these findings indicate that modulation of chronic stressinduced neuronal activity limits microglia-mediated neuronal remodeling in the medial PFC, and subsequent behavioral and cognitive consequences.
\end{abstract}

Neuropsychopharmacology (2020) 45:1766-1776; https://doi.org/10.1038/s41386-020-0720-1

\section{INTRODUCTION}

Stress-linked psychiatric disorders, such as major depressive disorder (MDD), are a significant contributor to global disease burden, leading to substantial socioeconomic costs [1, 2]. Evidence suggests that altered synaptic plasticity in corticolimbic brain regions, including the medial prefrontal cortex (PFC) underlie depressive symptoms [3,4]. The rodent model, chronic unpredictable stress (CUS), causes neuronal remodeling and synaptic deficits in the medial PFC that contribute to behavioral despair, anhedonia, and cognitive impairment [5-7]. In concert with neuronal remodeling, emerging data indicate that chronic stress alters microglial morphology and function [8, 9]. This is compelling as microglia can shape neuroplasticity through direct contact with neuronal structures (i.e., dendrites, synapses) and engulfment or trogocytosis of synaptic elements [10-12]. In this context, it is possible that stress-induced alterations in microglia contribute to the synaptic deficits underlying behavioral despair and cognitive impairment. Indeed our prior work indicates that CUS perturbs neuron-microglia interactions by increasing colonystimulating factor (CSF)-1 signaling in the medial PFC, provoking microglia-mediated neuronal remodeling and depressive-like behaviors [13, 14]. While these studies provide evidence that microglia contribute to stress effects on medial PFC structure and function, it remains unclear what neurobiological mechanisms promote CSF1 signaling and direct microglia-mediated neuronal remodeling.

Several lines of evidence indicate that stress causes aberrant neuronal activity in the PFC, which is thought to underlie synapse loss and associated behavioral consequences [6, 15-17]. Consistent with this idea, pharmacological interventions that dampen neuronal activity in the medial PFC have been shown to attenuate synaptic and behavioral deficits following chronic stress [18]. Benzodiazepines (i.e., diazepam) are a well-characterized drug class that acts on $\mathrm{GABA}_{\mathrm{A}}$ receptors to diminish excitatory neuronal activation [19, 20]. Indeed diazepam pre-treatment can dampen stress-evoked release of excitatory neurotransmitters in the medial PFC [21, 22]. In separate studies, diazepam is shown to attenuate anxiety- and depressive-like behaviors following stress exposure $[23,24]$. These neurophysiological responses are important because microglia are responsive to changes in neuronal activity, and reciprocally influence neuroplasticity through the release of neuromodulators (i.e., cytokines, growth factors) and interactions with synaptic elements [11, 12, 25]. Notably, microglia show increased surveillance and synaptic interactions in response to localized neuronal activity [26, 27]. In addition, other model systems show that elevated neuronal activity promotes CSF1 signaling. For example, kainic acid-induced neuronal hyperactivity increases CSF1 expression, and this is associated

${ }^{1}$ Department of Pharmacology \& Systems Physiology, University of Cincinnati College of Medicine, Cincinnati, OH, USA

Correspondence: Eric S. Wohleb (eric.wohleb@uc.edu)

Received: 19 February 2020 Revised: 27 April 2020 Accepted: 14 May 2020

Published online: 26 May 2020 
with microglial activation in the hippocampus [28]. Moreover, a recent study showed that optogenetic-induced neuronal activity increases Csf1 levels in the cortex and, in turn, promotes microglial phagocytosis of amyloid plaques in a model of Alzheimer's disease [29]. In this context, it is plausible that aberrant neuronal activity in response to chronic stress increases CSF1 signaling and microgliamediated neuronal remodeling, leading to synaptic deficits and associated behavioral consequences [13].

Expounding upon our previous findings [13], we used diazepam to test the role of stress-induced neuronal activation in microgliamediated neuronal remodeling in the PFC and associated behavioral or cognitive consequences. In addition to the PFC, we examined two brain regions implicated in stress responses (dorsal hippocampus, HPC; basolateral amygdala, BLA) as well as a brain region that is less responsive to stress (somatosensory cortex, SSCTX). Our results demonstrate that modulation of stressinduced neuronal activation with diazepam prevents behavioral and cognitive impairments, attenuates microglial phagocytosis of neuronal elements, and reduces synaptic deficits in the PFC. Notably, diazepam did not alter neuronal activation in the dorsal HPC, BLA, or the SSCTX, and expression of neuroimmune factors was not altered in the HPC following stress. Altogether, these results provide initial evidence that aberrant neuronal activity in response to chronic stress alters neuroimmune function in the PFC, and initiates microglia-mediated neuronal remodeling that contributes to synaptic deficits and associated behavioral consequences.

\section{MATERIALS AND METHODS}

\section{Animals}

Transgenic (and wild-type littermate) male Thy1-GFP(M) mice were obtained from in-house breeders (Jackson Laboratories; Tg (Thy1-EGFP)MJrs/J; \#007788). Behavioral and gene expression experiments were performed using male wild-type C57BL/6 mice (Jackson Laboratories; C57BL/6J; \#000664). Immunohistological experiments were conducted in Thy1-GFP(M) mice. All mice (6-8 weeks old) were group-housed (3-4/cage) in $11.5 \times 7.5 \times 6$ inch polypropylene cages under a 12-hour light-dark cycle with ad libitum access to food and water. Animal use and procedures were in accordance with the National Institutes of Health guidelines and approved by the University of Cincinnati Institutional Animal Care and Use Committees (IACUC).

Chronic unpredictable stress (CUS)

CUS was performed as previously described [13, 14]. Mice were exposed to random intermittent stressors over 14 days (2 stressors/day), including: cage rotation, isolation, restraint, radio noise, food or water deprivation, light on overnight, light off during day, rat odor, stroboscope overnight, crowding, wet bedding, no bedding, or tilted cage.

\section{Drug administration}

Diazepam (Millipore-Sigma; Saint Louis, Missouri, \#D0899) was diluted to $0.5 \mathrm{mg} / \mathrm{ml}$ in $0.9 \%$ sodium chloride $/ 0.1 \%$ ethanol. Diazepam was administered daily via intraperitoneal (i.p.) injection at $1 \mathrm{mg} / \mathrm{kg}$. To limit acute effects of drug treatments, injections were performed after behavioral testing in specified cohorts.

\section{Behavior and cognitive tests}

Forced swim test (FST), novelty-suppressed feeding test (NSFT), and temporal order recognition testing (TOR) were conducted as previously described [6, 13, 14]. All behavioral tests were performed in a normally lit room (white light), between 0800 and $1000 \mathrm{~h}$. Mice were allowed to habituate to this room for 30 min prior to testing. For the FST, mice were placed in a 2-liter beaker of water $\left(24^{\circ} \pm 1^{\circ} \mathrm{C}\right)$ for $10 \mathrm{~min}$ and immobility was measured. For the NSFT, mice were placed in a white plastic arena $(22 \times 30 \times 14 \mathrm{~cm})$ with fresh cage bedding covering the bottom. Three small pellets of chow were placed in the center of the arena and latency for the mice to feed was recorded. In the first trial phase of the TOR, mice were placed in a plastic arena with two plastic Lego ${ }^{T M}$ trees secured to the bottom of the arena. For each phase of the TOR, mice were given $5 \mathrm{~min}$ to explore the arena and objects. After a 30 min latency, mice were placed in the same arena with Lego ${ }^{\mathrm{TM}}$ blocks (second trial phase). An hour after the second trial phase, mice were placed in the arena with one block and one tree, counterbalanced to prevent bias (test phase). The time spent exploring each of these objects was measured and a discrimination index was calculated as follows: difference between time spent exploring the tree or the block divided by the total time spent exploring the tree and the block.

\section{RNA isolation and quantitative real-time PCR}

Whole PFC (medial frontal cortex between forceps minor prior to joining of corpus callosum) and HPC (dorsal and ventral) were rapidly dissected out $4 \mathrm{~h}$ after the final behavioral test. Total RNA was extracted from whole-brain regions using TRIzol Reagent according to manufacturer's protocol (Invitrogen). RNA was extracted from microglia using a Single Cell RNA purification kit (Norgen Biotek Corp., Thorold, Canada, \#51800). Samples were reverse transcribed, and quantitative real-time PCR was conducted as previously described $[13,14]$. Primer sequences are listed in Table S1.

Percoll gradient enrichment of microglia

Dissected frontal cortex (medial frontal cortex between forceps minor prior to joining of corpus callosum) was passed through a $70 \mu \mathrm{m}$ cell strainer. Homogenates were centrifuged at $1200 \times g$ for $5 \mathrm{~min}$. Supernatant was then removed and cell pellets were resuspended in 70\% isotonic Percoll (GE Healthcare, Uppsalla, Sweden, \#17089102). A discontinuous Percoll density gradient was layered as follows: 30 and $0 \%$ isotonic Percoll (phosphate-buffered saline, PBS). The gradient was centrifuged for $20 \mathrm{~min}$ at $2000 \times g$ and enriched microglia were collected from the interphase between the 70 and 30\% Percoll layers. Enriched microglia were then labeled with antibodies for flow cytometry.

Fluorescence-activated cell sorting (FACS)

Staining of cell surface antigens was performed as previously described [14]. In brief, Fc receptors were blocked with anti-CD16/ CD32 antibody (BioLegend, San Diego, CA, U.S.A., \#553141). Cells were washed and then incubated with conjugated antibodies (FITC-CX3CR1, \#149020; PE-CD45, \#103105; PE-Dazzle(564)-CD115, $\# 135528$; PerCp-Cy5-CD11b; BioLegend) for $1 \mathrm{~h}$ at $4{ }^{\circ} \mathrm{C}$. Cells were washed and re-suspended in fluorescence-activated cell sorting (FACS) buffer. Approximately 15,000 microglia were sorted based on $\mathrm{CD} 11 \mathrm{~b} / \mathrm{CD} 45$ expression using a BioRad S3e four-color cytometer/cell sorter (Hercules, CA, U.S.A.). Data were analyzed using FlowJo software (Ashland, OR, U.S.A.).

\section{Immunohistology}

Thy1-GFP(M) mice were transcardially perfused with sterile PBS and $4 \%$ paraformaldehyde (PFA) $4 \mathrm{~h}$ after the final stressor. Brains were post-fixed in $4 \%$ PFA for $24 \mathrm{~h}$ and incubated in $30 \%$ sucrose for an additional $24 \mathrm{~h}$. Frozen brains were sectioned on a Leica CM2050 S cryostat $(40 \mu \mathrm{m})$. Free-floating sections containing either the medial PFC (2.68-1.54 mm Bregma), BLA (-0.58$2.06 \mathrm{~mm}$ Bregma), or dorsal HPC and SSCTX $(-1.06-2.30 \mathrm{~mm}$ Bregma) were selected for immunohistology. Sections were washed, blocked for $1 \mathrm{~h}$ at room temperature, washed, and then incubated with primary antibody: rabbit anti-IBA1 (1:1000, Wako; 019-19741) or rabbit anti-FosB (1:1000, Abcam; ab184938) overnight at $4{ }^{\circ} \mathrm{C}$. Sections were then washed and incubated with conjugated secondary antibody overnight at $4{ }^{\circ} \mathrm{C}(1: 1000$, Invitrogen; Alexa Fluor 488, A21206; Alexa Fluor 546, A10040). 
Quantitative immunofluorescence. Confocal images were obtained on a Nikon C2+ microscope interfaced with a Nikon $\mathrm{C} 2 \mathrm{si}+$ camera. Quantification of immunolabeling and dendritic spine density was performed as previously described using ImageJ and NeuronStudio software $[13,30]$. Confocal images were captured from adjacent brain sections in both hemispheres of each region of interest (3-4 sections/sample). All regions were identified using structural landmarks within Bregma coordinates listed above. To quantify FosB + cells, immunolabeled cells were counted using the ImageJ count function (circularity: 0.2-1, size: 30-300) for all brain regions except BLA. Given its unique shape and the abundance of nearby nuclei, the BLA was traced and measured in ImageJ. Cells were counted by a trained researcher in this region. Cell counts were standardized to image- (medial PFC, dorsal HPC, SSCTX) or region- size (BLA) and total FosB + cell density was calculated (cells per $\mathrm{mm}^{2}$ ). For microglia analyses (IBA1): individual cell counts were obtained on standardized images and cell density was calculated (cells per $\mathrm{mm}^{2}$ ). For proportional area images were thresholded and total area was recorded (IBA1 \% area). In Thy1-GFP(M) mice, layer I of the medial PFC was identified in multiple adjacent sections and apical dendritic segments were imaged using $40 x$ objective lens (NA 0.95; zoom 2.5×, z-stack: $0.1 \mu \mathrm{m})$. Images were analyzed using NeuronStudio (6-8 segments/ sample) as previously described [13]. To quantify GFP + inclusions, confocal images were obtained in multiple adjacent sections and microglia with complete morphological profiles were identified (14-24 cells/sample). Confocal images (40x; zoom 2.5×, NA 0.95, z-stack: $0.1 \mu \mathrm{m}$ ) were examined in 3-dimensional space and orthogonal z-stacks with Nikon Elements Image Analysis software. Confocal imaging with these settings provides sufficient resolution to detect synaptic inclusions (average inclusion diameter: $0.25 \mu \mathrm{m}$ ) [12].

Statistical analysis. Data were analyzed using GraphPad Prism 8.1.2 (La Jolla, California). Significant main effects and interactions were determined using two-way ANOVA (stress $\times$ drug treatment). Differences between group means were evaluated with Tukey's multiple comparisons test. The linear relationship between behavioral measures and gene expression analyses, and histological measures was examined using Pearson's correlation coefficient. One outlier was identified using the Grubb's method and was subsequently removed from analysis (microglial CD68 mRNA, control-diazepam sample). The number of animals examined in each analysis are noted in Table S2.

\section{RESULTS}

Diazepam reduced neuronal activation in the prefrontal cortex and prevented behavioral despair and working memory deficits following CUS

Prior work indicates that the $\mathrm{GABA}_{\mathrm{A}}$ receptor modulator diazepam reduces neuronal activity in the medial PFC, and may attenuate stress-induced cognitive and behavioral deficits [23, 31]. To assess this, we analyzed FosB levels in the medial PFC in vehicle- or diazepam-treated mice following CUS (Fig. 1a). Stress exposure caused a significant increase in FosB + cells $/ \mathrm{mm}^{2}$ in the medial PFC, and treatment with diazepam blocked this effect (interaction: $F_{1,19}=7.59, p=0.01$; Fig. 1b, f). Post-hoc analyses confirmed that CUS increased the number of FosB + cells in vehicle-treated animals as compared to all other groups ( $p \leq 0.02$, Fig. $1 f)$. To determine the brain region-specificity of these effects, the CA1 region of the dorsal HPC, BLA, and SSCTX was examined. These brain regions were selected because they express $G_{A B A}$ receptors, and both the BLA and the CA1 region of dorsal HPC show altered function in chronic stress [32-34], whereas neuronal function in the SSCTX and SSCTXassociated behaviors are not significantly altered [35]. There were no effects of stress or diazepam treatment on the number of FosB + cells $/ \mathrm{mm}^{2}$ in the CA1 region of dorsal HPC (Fig. 1c, f), BLA (Fig. 1d, f), or SSCTX (Fig. 1e, f).
In a separate cohort, mice were assessed for forced swim test immobility (FST), novel environment aversion (NSF), and working memory performance (TOR; Fig. 2a). CUS exposure increased immobility in the FST, whereas diazepam administration blocked this effect (interaction: $F_{1,37}=4.53, p=0.04$; Fig. 2b). Likewise, CUS mice had increased latency to feed in the NSF, and this effect was attenuated by diazepam (interaction: $\mathrm{F}_{1,34}=5.73, p=0.02$; Fig. $2 \mathrm{c}$ ). In addition, mice exposed to CUS showed discrimination deficits in the TOR, and diazepam prevented these cognitive impairments (interaction: $\mathrm{F}_{1,28}=7.69, p=0.01$; Fig. $2 \mathrm{~d}$ ).

Following behavioral testing, both PFC and HPC were dissected to examine gene expression of factors associated with microglianeuron interactions and neuroimmune function. Mice exposed to CUS had increased expression of Csf1 in the PFC, and diazepam normalized Csf1 levels (interaction: $\mathrm{F}_{1,28}=6.64, p=0.01$; Fig. 2e). Analyses indicate that $C \times 3 \mathrm{Cl} 1$ transcript levels in the PFC varied with CUS exposure and diazepam administration (interaction: $\mathrm{F}_{1,28}=6.41, \quad p=0.02$ ), however, no significant differences emerged with post-hoc comparisons (Fig. 2e). Levels of Tgfb1 mRNA differed with diazepam treatment (drug: $\mathrm{F}_{1,28}=4.45, p=$ $0.04)$, but not stress. There were no effects of diazepam or stress on I/34 transcript expression in the PFC of mice. Expression of Tnfa in the PFC differed with both CUS exposure (stress: $F_{1,28}=3.45$, $p=0.07$ ) and diazepam treatment (drug: $\mathrm{F}_{1,28}=13.08, p=0.001$ ). Post-hoc comparisons suggest that these effects were driven by modest increases in Tnfa in both control $(p=0.10)$ and CUS $(p=$ 0.05 ) mice treated with diazepam (Fig. 2e). Mice exposed to CUS also had increased C1q transcript in the PFC (stress: $\mathrm{F}_{1,28}=28.44$, $p<0.0001$ ), but diazepam did not affect this response (Fig. 2e). There was a significant effect of CUS on C3 expression, and diazepam attenuated this stress-induced increase (interaction: $\mathrm{F}_{1,28}=7.39, p=0.01$ ).

Expression levels of specific neuroimmune factors in the medial PFC correlated with behavioral performance. Increased expression of Csf1 was associated with greater immobility in the FST $\left(r_{(30)}=\right.$ $0.36, p=0.04$; Fig. S1A), increased latency to feed in the NSFT $\left(r_{(27)}=0.55, p=0.002\right.$; Fig. S1B), and dysfunction in the TOR $\left(r_{(30)}=-0.54, p=0.002\right.$; Fig. S1C). Likewise, heighted expression of $C 1 q$ and $C 3$ was associated with deficits in the NSFT (C1q: $r_{(27)}=$ $0.49, p=0.007$; C3: $r_{(27)}=0.42, p=0.02$; Fig. S1B) and TOR (C1q: $r_{(30)}=-0.37, p=0.04 ;$ C $3: r_{(30)}=-0.40, p=0.02$; Fig. S1C). Decreases in Tnfa were associated with poorer working memory performance in the TOR $\left(r_{(30)}=0.41, p=0.02\right.$; Fig. S1C). Neither II34, Cx3cl1, nor Tgfb1 correlated with behavioral function.

In the HPC, stress effects on both Csf1 and I/34 expression varied with diazepam treatment ( $C s f 1$ interaction: $F_{1,27}=5.00, p=0.03$; II34 interaction: $\mathrm{F}_{1,26}=4.72, p=0.04$; Fig. 1g), however, post-hoc analyses revealed no significant group differences for either gene. Transcript levels of $\mathrm{C} \times 3 \mathrm{Cl} 11$ differed with diazepam treatment $\left(\mathrm{F}_{1,27}=6.11, p=0.02\right)$, but not stress. There were no effects of diazepam or stress on Tgfb1 expression. Levels of Tnfa mRNA were affected by CUS $\left(F_{1,25}=4.71, p=0.04\right)$, regardless of diazepam administration. There were no effects of diazepam or stress on $\mathrm{Cl} q$ or $\mathrm{C} 3$ expression in the HPC. In contrast to the medial PFC, greater expression of Csf1 in the HPC was associated with reduced immobility in the FST $\left(r_{(29)}=-0.41, p=0.02\right.$; Fig. S2A). Heightened expression of $1 / 34$ was also associated with reduced immobility in the FST $\left(r_{(28)}=-0.41, p=0.02\right.$; Fig. S2A), alongside reduced latency to feed in the NSFT $\left(r_{(26)}=-0.41, p=0.03\right.$; Fig. $\mathrm{S} 2 \mathrm{~B})$. Likewise, increased Tgfb1 was associated with decreased immobility in the FST $\left(r_{(28)}=-0.53, p=0.003\right.$; Fig. S2A). Neither $C \times 3 C_{1}, C 19, C 3$, nor Tnfa correlated with behavioral measures, nor were any genes in the HPC associated with performance in the TOR (Fig. S2C). These studies indicate that diazepam reduced CUSinduced neuronal activation in the medial PFC, attenuated behavioral and cognitive impairments, and normalized expression of select neuroimmune factors in the PFC. In comparison, the HPC was largely unaffected by CUS. Moreover, correlational analyses 
A.

CUS (14 days)
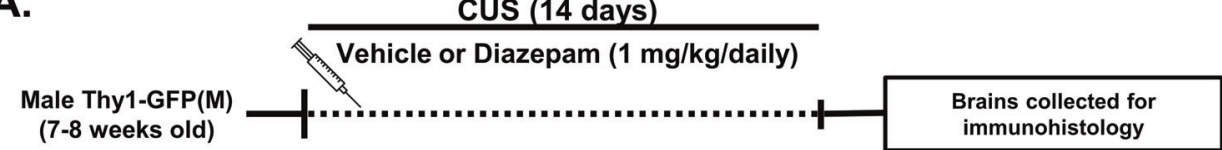

B.

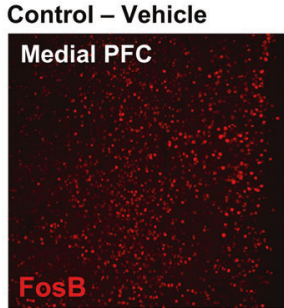

Control - Diazepam

CUS - Vehicle
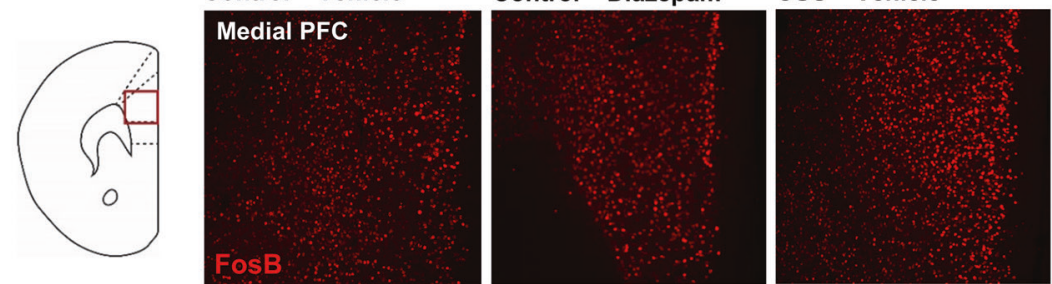

CUS - Diazepam

C.
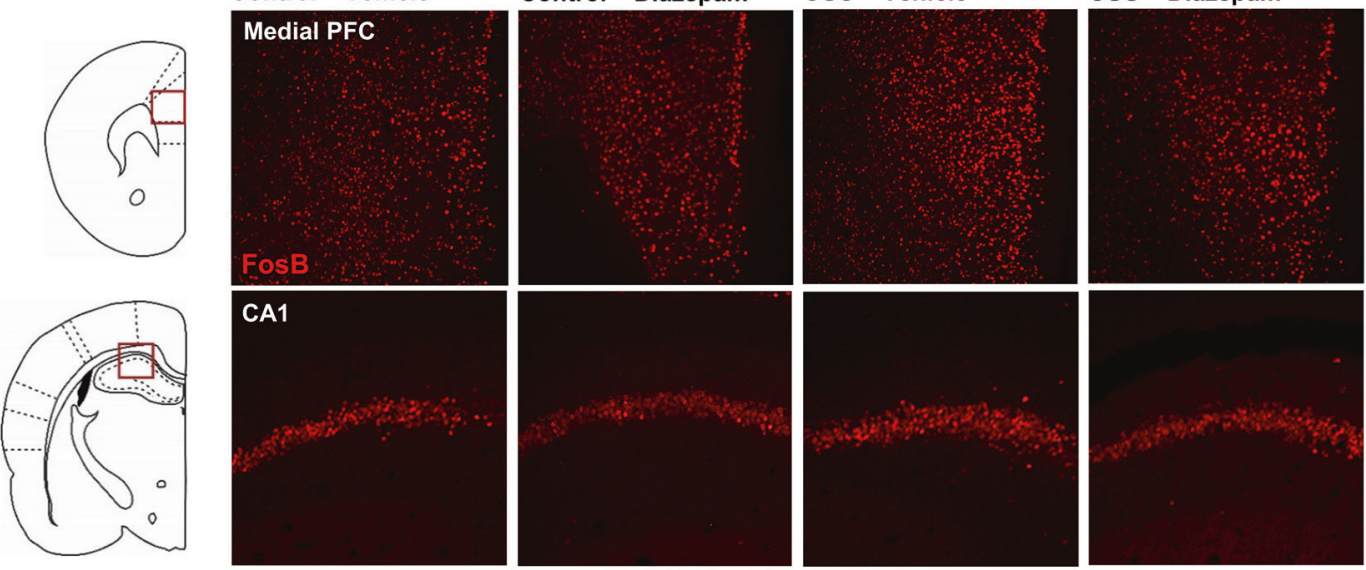

D.
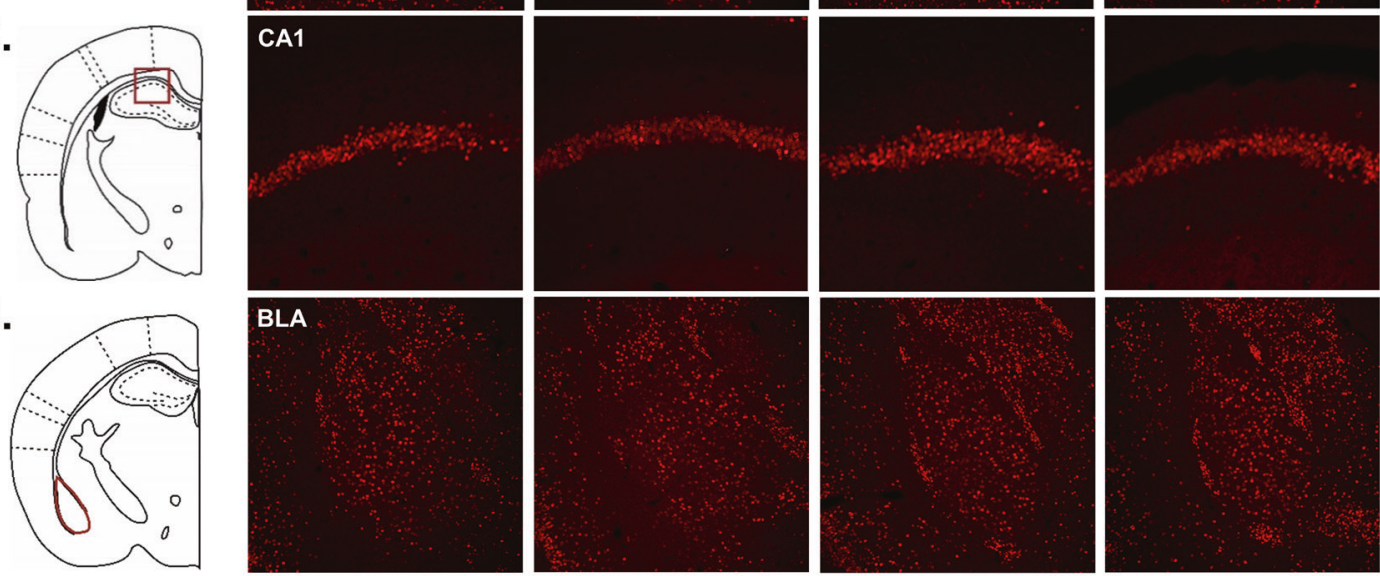

E.
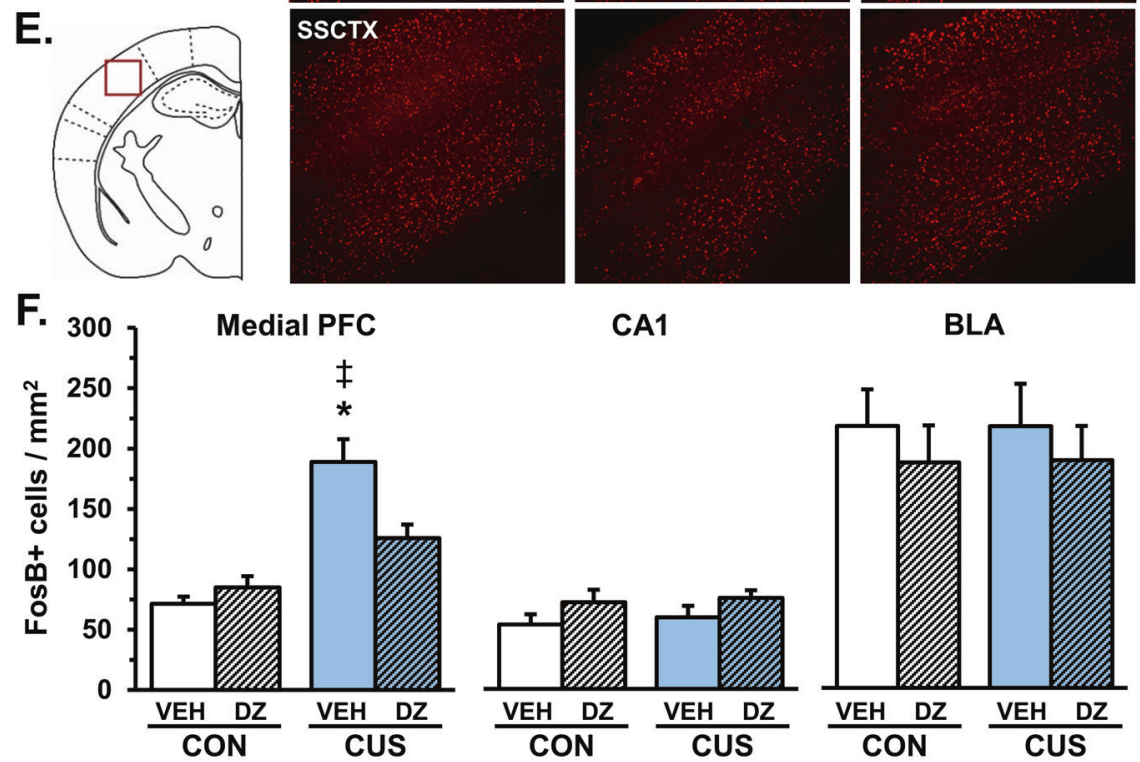

CA1

BLA
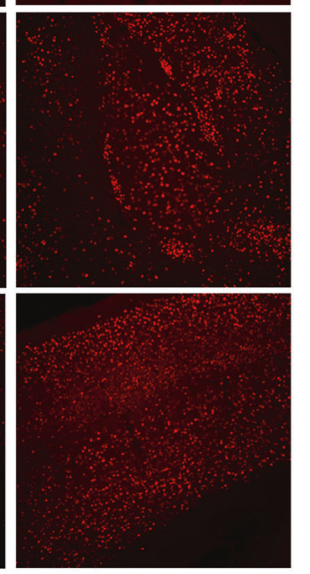

SSCTX

Fig. 1 Diazepam attenuates stress-induced increases in neuronal activation in the PFC. a Male C57BL/6 J mice were exposed to 14 days of CUS or handled intermittently as controls. Mice received daily injection of either vehicle or diazepam (1 mg/kg, i.p.). Following CUS, animals were perfused, and brains extracted and processed for immunohistology. Left: Schematic representation of the b medial PFC, c CA1 region of the dorsal HPC, d BLA, and e the SSCTX directly lateral to the dorsal HPC. One hemisphere shown for simplicity. Right: Representative images of FosB immunolabeling in each region sampled. $\mathbf{f}$ Left to right: Average number of FosB + cells/ $\mathrm{mm}^{2}$ in the medial PFC, CA1 region of the dorsal HPC, BLA, and the somatosensory cortex (right; $n=5-8 /$ group). Bars represent mean \pm S.E.M. * denotes $p<0.05$, compared to respective control group. $\neq$ denotes $p<0.05$, compared to CUS-diazepam group.

indicated a stronger link between neuroimmune gene expression in the medial PFC and behavior, particularly working memory function. As such, the effects of diazepam on microglial function and microglia-neuron interaction following CUS were examined in the PFC.
Diazepam partially attenuated chronic stress effects on the molecular profile of frontal cortex microglia

To further study the effects of diazepam on stress-induced neuroimmune activity in the PFC, microglia were enriched from frontal cortex via Percoll gradient and then purified via 
A.

CUS (14 days)
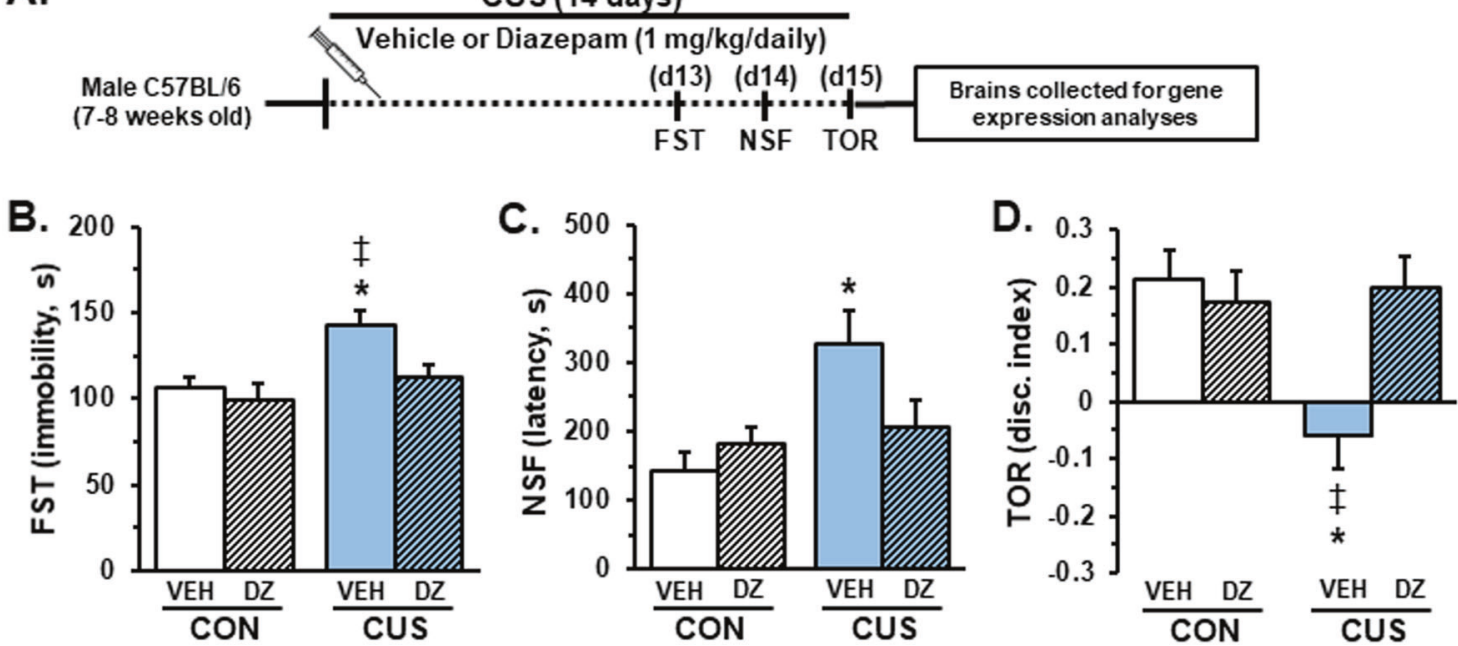

\section{E.}
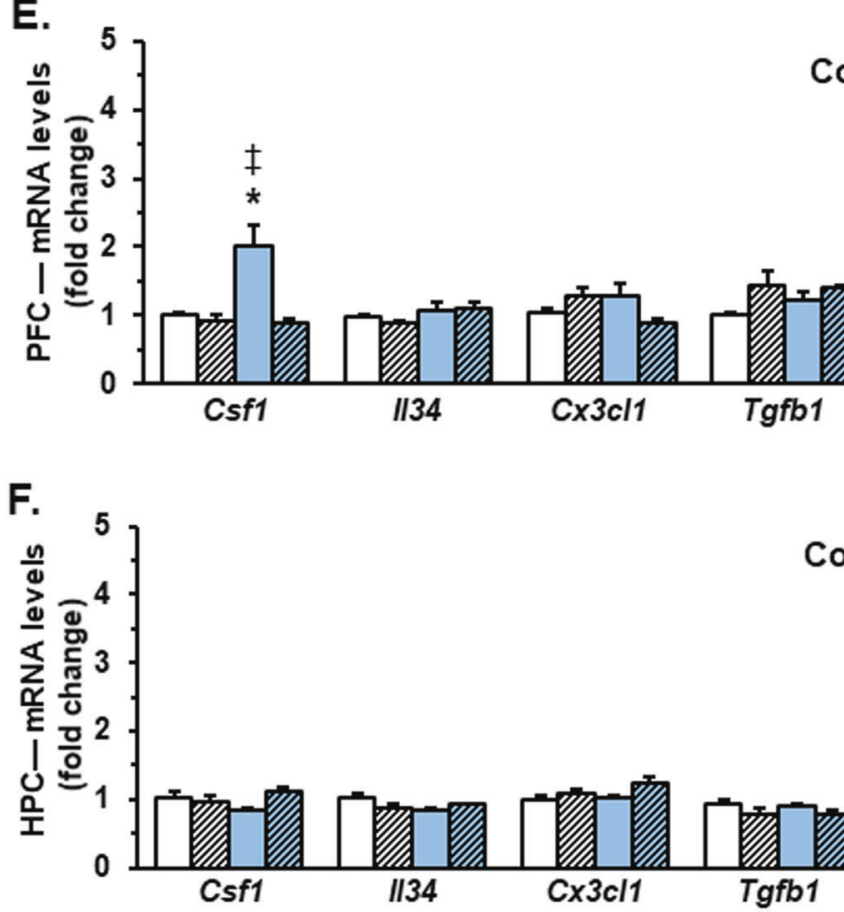

Control $\mid \square$ Vehicle

cus

Vehicle Diazepam

Fig. 2 Diazepam prevents stress effects on behavior, cognitive function, and neuroimmune gene expression in the PFC. a Male C57BL/6 J mice were exposed to 14 days of CUS or handled intermittently as controls. Mice received daily injection of either vehicle or diazepam (1 mg/ $\mathrm{kg}$, i.p.). Forced swim test (FST), novelty-suppressed feeding (NSF), and temporal object recognition (TOR) were assessed on subsequent days ( $n=8-11$ /group). b Immobility in the forced swim test (FST), c Latency to feed in the novelty-suppressed feeding test (NSF), and d Discrimination index in the temporal object recognition (TOR) is presented. Two hours after the final behavioral assay, e PFC and $\mathbf{f}$ HPC were dissected for gene expression analyses. Relative fold change in mRNA levels is shown ( $n=6-8 / g r o u p)$. Bars represent mean \pm S.E.M. * denotes $p<0.05$, compared to respective control group. $\neq$ denotes $p<0.05$, compared to CUS-diazepam group.

fluorescence-activated cell sorting (FACS) (Fig. 3a). Representative dot plots show the distribution of microglia $\left(\mathrm{CD} 11 \mathrm{~b}^{+} / \mathrm{CD} 45^{\mathrm{lo}}\right)$ and brain macrophages $\left(C D 11 b^{+} / C D 45^{\text {hi }}\right)$ from the frontal cortex (Fig. 3b). Of note, the proportion of microglia and brain macrophages were not significantly altered by CUS or diazepam treatment (Fig. 3c). Further analyses showed that CUS increased CSF1R (CD115) surface expression on microglia (mean fluorescence intensity $[\mathrm{MFI}],+7.5 \%$ ) (interaction: $\mathrm{F}_{1,25}=4.48, p=0.04$ ), however post-hoc comparisons did not reveal significant group differences (Fig. 3d). Moreover, surface expression of CX3CR1 on microglia was unchanged (Fig. 3e), but CUS increased CD11b levels on microglia $(\mathrm{MFI},+8.3 \%)$, and this effect was prevented by diazepam administration (interaction: $F_{1,25}=6.19, \quad p=0.02$; Fig. 3f).

Following FACS, gene expression of select factors were assessed in microglia. As expected, CUS caused a robust increase in microglial Csf1r expression, and treatment with diazepam blocked this effect (interaction: $\mathrm{F}_{1,26}=7.63, p=0.01$; Fig. $3 \mathrm{~g}$ ). In contrast, microglial $\mathrm{C} \times 3 \mathrm{Cr} 1$ expression was elevated after CUS (stress: $\mathrm{F}_{1,25}=6.15, p=0.02$ ), but did not vary with diazepam treatment and no significant post-hoc differences were evident (Fig. $3 g$ ). There were no changes observed in microglial Tgfbr 1 expression. Further, CUS increased microglial $C d 11 b$ expression (stress: $F_{1,28}=$ 7.00, $p=0.01$ ), and diazepam treatment blocked this response 
A.

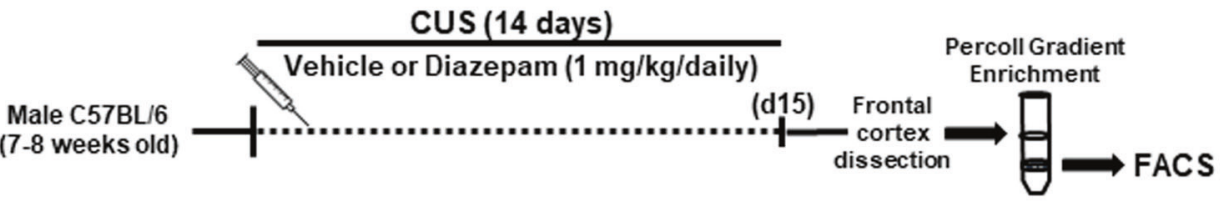

B.

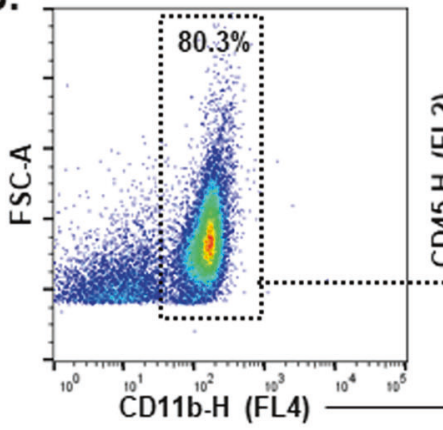

D.

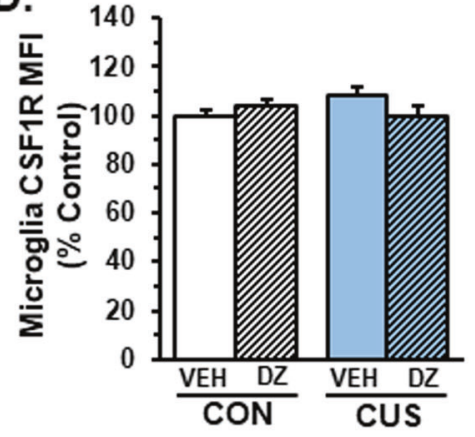

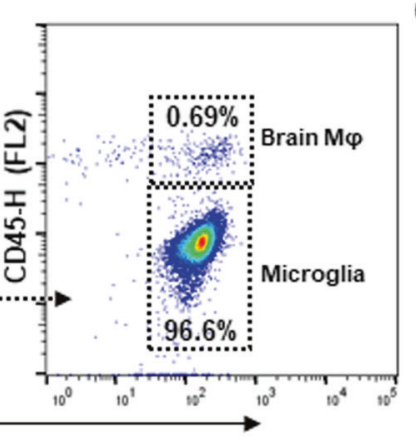

E.

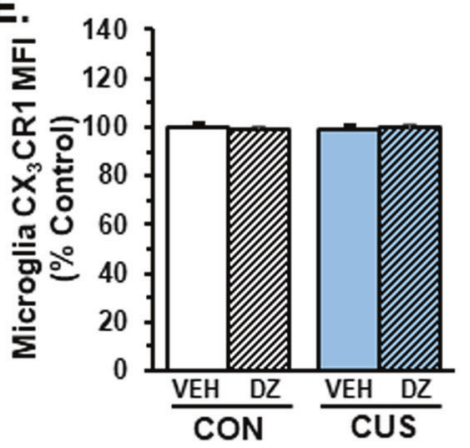

C.

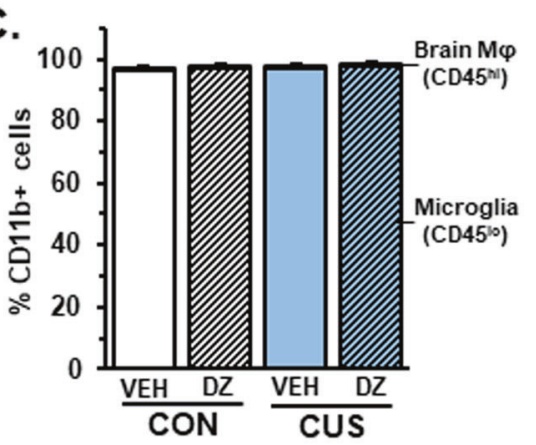

F.

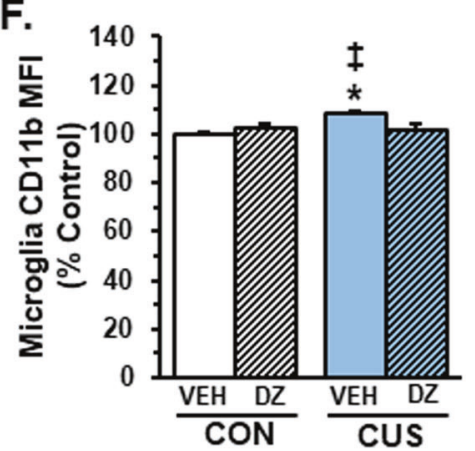

G.

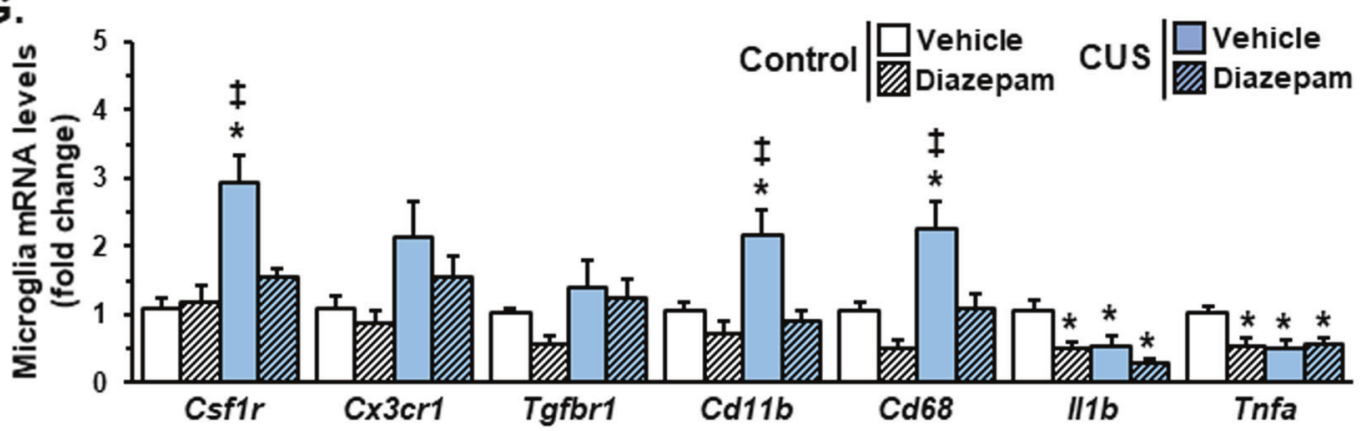

Fig. 3 Diazepam attenuates stress effects on microglial gene expression in the PFC. a Male C57BL/6 J mice were exposed to 14 days of chronic unpredictable stress (CUS) or handled intermittently as controls. Mice received daily injection of either vehicle or diazepam (1 mg/kg, i.p.). Two hours after the final stressor, frontal cortex was dissected and brain myeloid cells were enriched via Percoll gradient. Fluorescenceactivated cell sorting (FACS) was used to isolate microglia based on CD $11 b^{+} / C D 45^{\text {lo }}$ expression. $\mathbf{b}$ Representative dot plots of FSC/CD11b and CD45/CD11b in samples. c Average proportion of microglia (CD $11 \mathrm{~b}^{+} / \mathrm{CD}_{4} 5^{\mathrm{lo}}$ ) and brain macrophages $\left(\mathrm{CD} 11 \mathrm{~b}^{+} / \mathrm{CD} 45^{\mathrm{hi}}\right)$ in each experimental group. Normalized mean fluorescent intensity is shown for d CSF1R (CD115), e CX ${ }_{3}$ CR1, and f CD11b (CR3). Following FACS, mRNA was collected from microglia. g Relative fold change in mRNA levels in sorted frontal cortex microglia is shown ( $n=6-8 /$ group). One outlier was removed from analysis of microglial Cd68. Bars represent mean \pm S.E.M. * denotes $p<0.05$, compared to respective control group. $\neq$ denotes $p<0.05$, compared to CUS-diazepam group.

(drug: $F_{1,28}=10.52, p=0.003$ ). Post-hoc comparisons showed that microglia from CUS-vehicle mice had significantly higher levels of $C d 11 b$ transcript compared to all other groups ( $p=0.018$; Fig. $3 \mathrm{~g}$ ). Similarly, CUS increased microglial expression of Cd68 (stress: $F_{1,27}=10.70, p=0.003$ ), and diazepam attenuated this effect (drug: $F_{1,28}=9.44, p=0.005$ ). Microglial $/ 11 b$ expression differed with stress $\left(\mathrm{F}_{1,25}=11.46, p=0.002\right)$ and diazepam treatment $\left(F_{1,25}=9.95, p=0.004\right.$; Fig. $\left.3 g\right)$. In addition, CUS and diazepam administration caused a robust decrease in microglial Tnfa expression (interaction: $\mathrm{F}_{1,26}=6.73, p=0.015$; Fig. 3g). Altogether these results demonstrate that diazepam partially attenuates chronic stress-induced molecular adaptations in frontal cortex microglia.

Diazepam blocked chronic stress-induced dendritic spine loss and microglia-mediated neuronal remodeling in the medial PFC

In the same tissue used for FosB immunohistology (Fig. 1b), confocal imaging was performed to examine the morphological features of IBA1 + microglia and dendritic spine density of Thy1GFP(M) pyramidal neurons in the medial PFC. Neither diazepam treatment nor CUS exposure affected the number of IBA1 + cells/ $\mathrm{mm}^{2}$ or the proportional area of IBA $1+$ microglia in the medial 

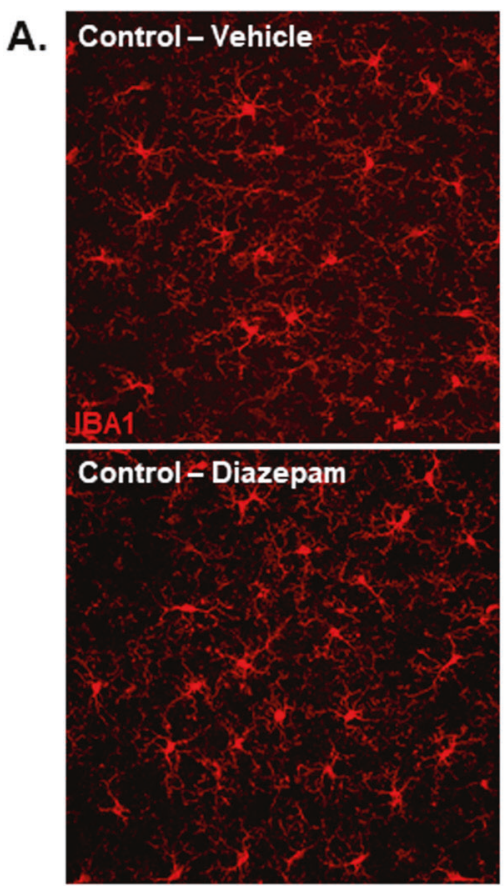

D.
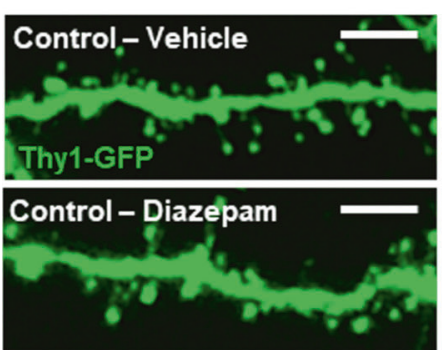
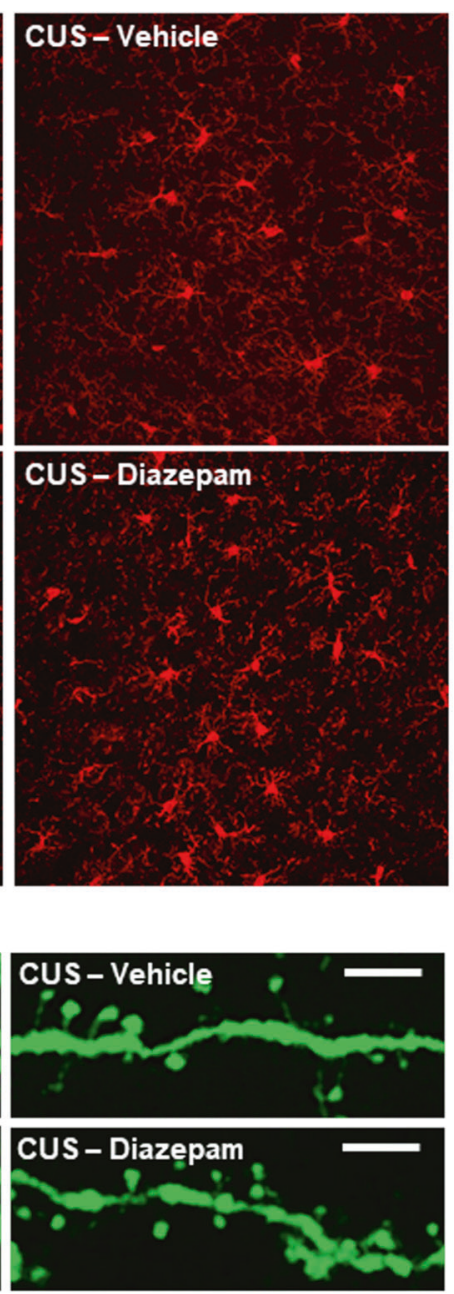
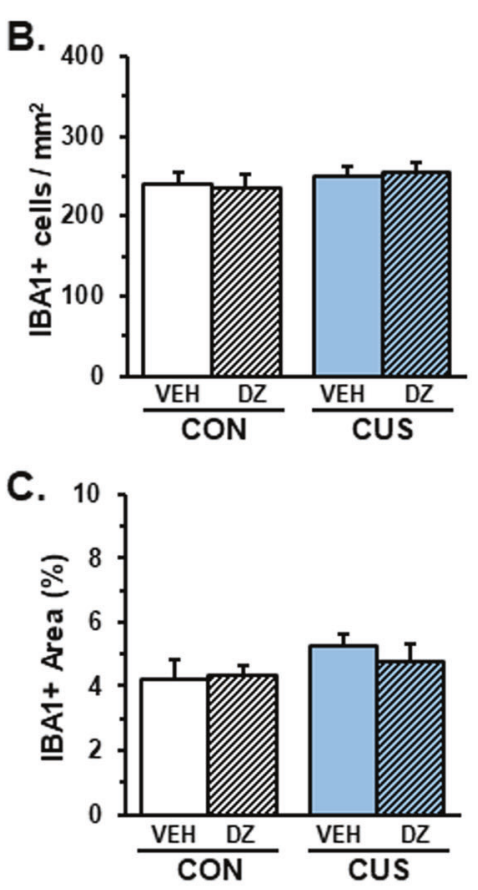

E.

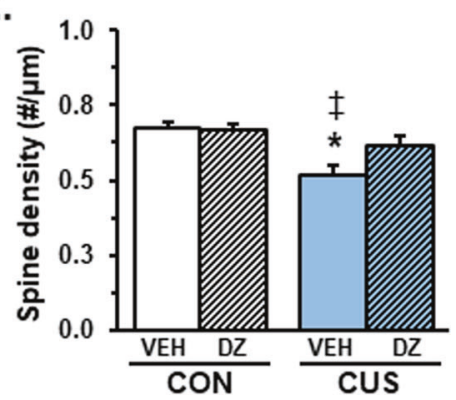

Fig. 4 Chronic stress-induced dendritic spine deficits are blocked by diazepam administration. Male Thy1-GFP(M) mice were exposed to 14 days of chronic unpredictable stress (CUS) or handled intermittently as controls. Mice received daily injection of either vehicle or diazepam $(1 \mathrm{mg} / \mathrm{kg}$, i.p.). The same brains used for FosB immunolabeling in Fig. 1 were used for IBA1 immunohistology and dendritic spine analyses. a Representative images of IBA1 immunofluorescence in the medial PFC of all experimental groups is shown. $\mathbf{b}$ Average number of IBA $1+$ cells $/ \mathrm{mm}^{2}$ in the medial PFC, and c Quantification of IBA1 proportional (\%) area in the medial PFC is presented ( $n=6-9 / \mathrm{group}$ ). d Representative confocal images of layer I apical dendrites in the medial PFC of each experimental group. White scale bar represents $5 \mu \mathrm{m}$. e Quantification of average dendritic spine density is shown ( $n=5-6 /$ group). Bars represent mean \pm S.E.M. * denotes $p<0.05$, compared to respective control group. $\neq$ denotes $p<0.05$, compared to CUS-diazepam group.

PFC (Fig. 4a-c). However, stress-reduced dendritic spine density on the apical dendrites of pyramidal neurons in the medial PFC in vehicle - but not diazepam - treated mice (interaction: $F_{1,17}=$ 5.18, $p=0.04$; Fig. 4d, e). Increased FosB + cell density correlated with dendritic spine loss in the medial PFC $\left(r_{(15)}=-0.72, p=\right.$ 0.001; Fig. S3).

To further examine neuron-microglia interactions, confocal images were collected in lamina I of the medial PFC of Thy1GFP(M) mice. These analyses revealed that CUS increased the proportion of microglia with GFP + inclusions in the medial PFC, and microglial engulfment of GFP + inclusions was limited by treatment with diazepam (interaction: $F_{1,17}=10.34, p=0.005$; Fig. 5a, b, Fig. S4). Further analyses showed that CUS increased the number of GFP + inclusions per microglia, and this effect was attenuated by diazepam (interaction: $F_{1,17}=14.45, p=0.001$; Fig. 5c). Likewise, CUS increased the total volume of GFP + inclusion per microglia, and diazepam prevented this response (interaction: $\mathrm{F}_{1,17}=12.46, p=0.003$; Fig. $5 \mathrm{~d}$ ). Heightened FosB+ cell density in the medial PFC was associated with increased microglial engulfment of GFP + inclusions $\left(r_{(15)}=0.66, p=0.004\right.$; Fig. S3), a greater number of GFP + inclusions per microglia $\left(r_{(15)}=0.53, p=0.03\right.$; Fig. S3), and increased volume of GFP + inclusions per microglia $\left(r_{(15)}=0.74, p<0.001\right.$; Fig. S3). All measures of microglial engulfment were correlated with dendritic spine loss in the medial PFC (proportion of microglia with inclusions: $r_{(19)}=-0.69, p<0.001$; number of inclusions per microglia: $r_{(19)}=-0.72, p<0.001$; volume of inclusions: $r_{(19)}=$ $-0.70, p<0.001$; Fig. S3). Altogether these results provide evidence that diazepam limited microglia-mediated neuronal remodeling following CUS, which was linked to attenuated spine deficits in the medial PFC.

\section{DISCUSSION}

Clinical and preclinical studies indicate that stress exposure plays a role in the etiology of several psychiatric diseases, and promotes pathophysiological changes in the brain including synapse loss in the medial PFC [36-38]. In particular, chronic stress leads to significant reductions in dendritic length and dendritic spine density on apical (lamina I) - but not basilar (lamina V) - dendritic branches in the medial PFC $[38,39]$. Though intrinsic pathways in neurons have been identified $[7,40]$, recent findings indicate that 

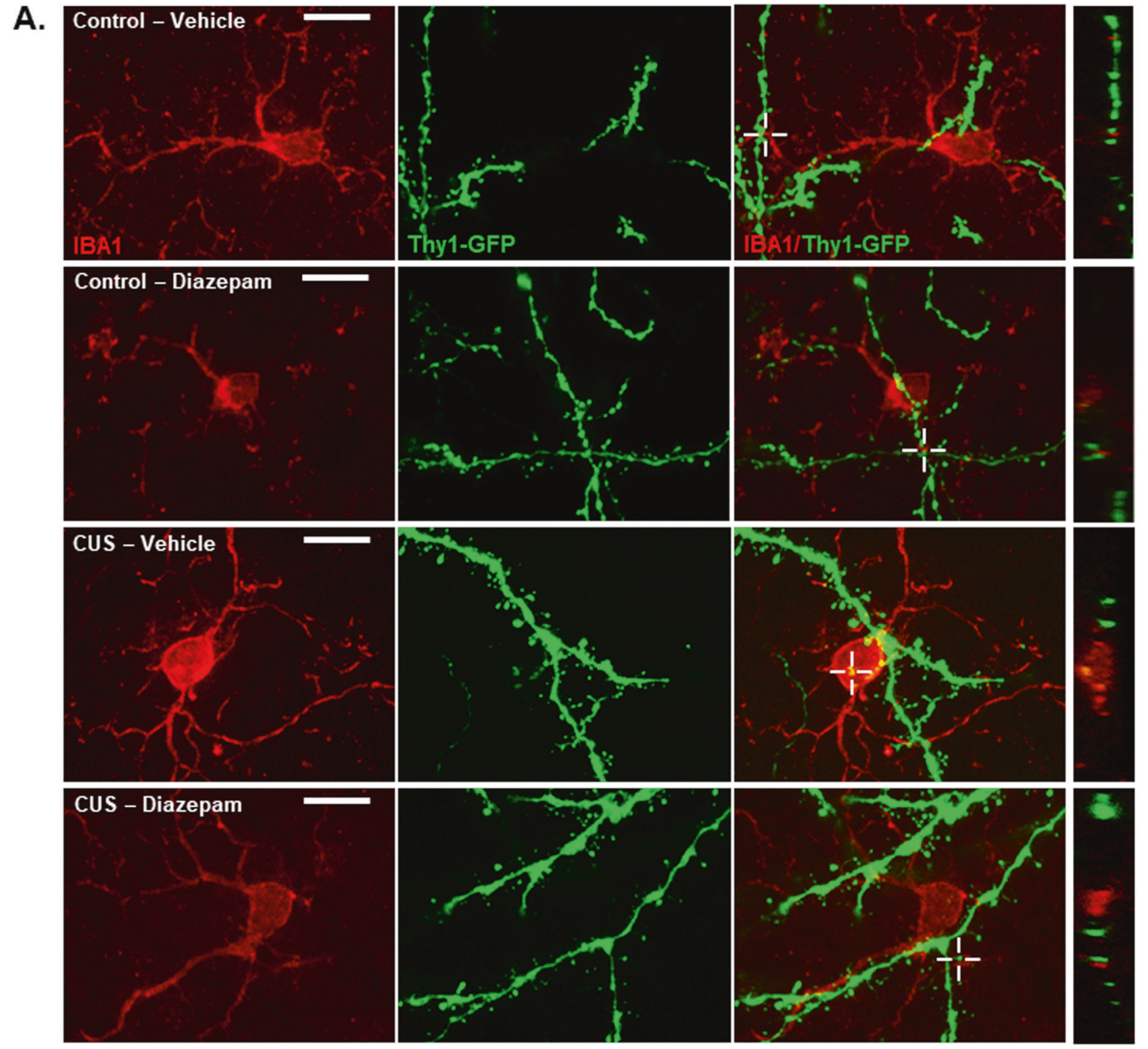

B.

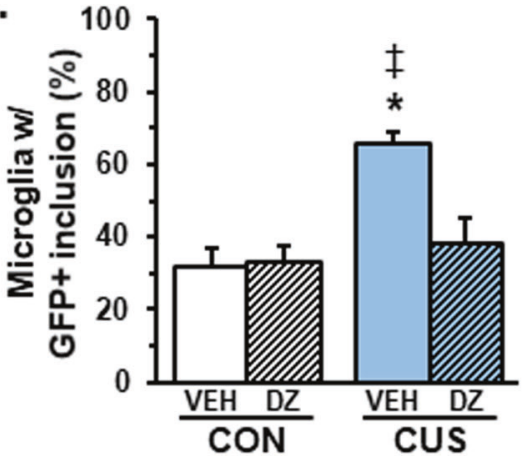

C.

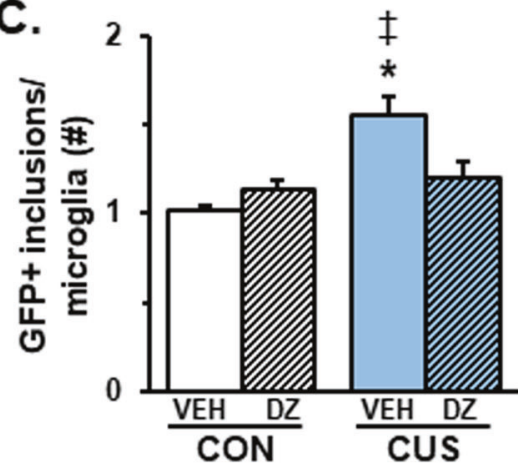

D.

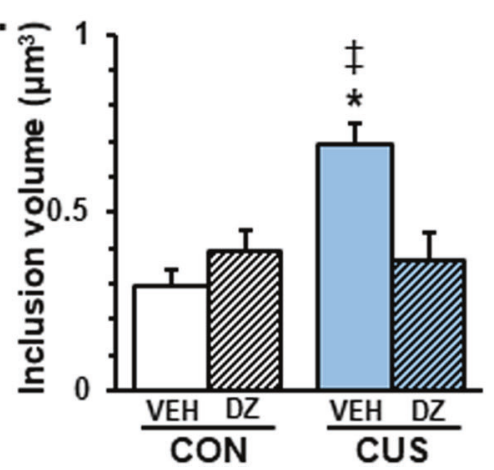

Fig. 5 Diazepam limited microglia-mediated neuronal remodeling in layer I of the medial PFC following chronic stress. Male Thy1-GFP(M) mice were exposed to 14 days of chronic unpredictable stress (CUS) or were handled intermittently as controls. Mice received daily injection of either vehicle or diazepam (1 mg/kg, i.p.). The same brains used for FosB immunolabeling in Fig. 1 were used for analyses of neuronmicroglia interactions. a Confocal images of individual and merged channels obtained from lamina I of the medial PFC in control or CUS mice treated with vehicle or diazepam. The last panel (right) shows an orthogonal cross-section at designated coordinates. White scale bar represents $10 \mu \mathrm{m}$. b Proportion of microglia in layer I of medial PFC with GFP + inclusions. c Average number of GFP + inclusions in microglia with neuronal elements. d Total volume of inclusions $\left(\mu \mathrm{m}^{3}\right)$ per microglia in layer I of the medial PFC $(n=5-6 /$ group$)$. Bars represent mean \pm S.E.M. * denotes $p<0.05$, compared to respective control group. $\neq$ denotes $p<0.05$, compared to CUS-diazepam group. 
1774

dynamic microglia-neuron interactions contribute to stressinduced neuronal remodeling in the medial PFC $[13,41]$. Indeed microglia can modulate synaptic activity through release of signaling molecules and can contribute to structural remodeling of neurons through phagocytosis of pre- and post- synaptic elements $[11,25]$. Of note, our recent work shows that chronic stress drives microglial phagocytosis of neuronal elements in lamina I - but not lamina V - of the medial PFC and this contributes to reductions in apical dendritic spine density $[13,14]$. Here we expound on these studies providing initial evidence that increased microglia-mediated neuronal remodeling and associated behavioral consequences following chronic stress are mediated, in part, by neuronal activity in the medial PFC.

In the present study, diazepam was used to pharmacologically block chronic stress-induced neuronal activation in the medial PFC. Diazepam is a benzodiazepine that enhances the inhibitory function of $\mathrm{GABA}_{A}$ receptors, thereby reducing the excitability of pyramidal neurons [42]. Indeed prior studies show that diazepam reduces neuronal activity in corticolimbic circuitry and these neurophysiological effects are associated with reduced arousal and attenuated stress effects on behavior $[19,43]$. Consistent with these reports, our results showed that diazepam reduced FosB+ cells in the medial PFC, and this effect was associated with diminished behavioral and cognitive consequences following CUS exposure. It is relevant to note that these analyses are limited as FosB immunolabeling is an indirect measure of neuronal activity. Moreover, our studies used peripheral administration of diazepam. Though stress and diazepam did not affect FosB levels in other corticolimbic brain regions (i.e., CA1 of the dorsal HPC, BLA), we cannot rule out the possibility that other corticolimbic brain regions contribute to the neurobiological and behavioral effects of diazepam. Future work can utilize more direct approaches to manipulate PFC function (i.e., chemogenetics) and to assess circuit-specific changes in neuronal activity (i.e., patch-clamp electrophysiology or fiber photometry). In all, our findings lend support to studies connecting stress-associated neuronal activity in the medial PFC with subsequent behavioral consequences $[15,18]$.

One novel finding in this study is that modulation of neuronal activation via diazepam influences neuron-microglia interactions in the medial PFC. In particular, diazepam diminished stressinduced increases in Csf1 transcript in the medial PFC, and reduced expression of Csfir in sorted microglia following chronic stress. Several studies show that CSF1 signaling is critical in regulating microglial homeostasis and survival $[44,45]$. Moreover, our prior work indicates that CSF1 signaling provokes functional alterations in microglia following chronic stress [13, 14]. Interestingly, other studies demonstrate that increased neuronal activity can induce CSF1 signaling and microglia activation in different models of neurological disease $[28,29]$. Our findings also show that chronic stress increased $\mathrm{C} 1 q$ and $\mathrm{C} 3$ expression in the medial PFC, alongside heightened expression of CD11b transcript and surface protein on microglia. Notably, diazepam normalized expression of these complement factors in the medial PFC, and reduced microglial $C D 11 \mathrm{~b}$ expression following chronic stress. This is important because recent studies show that the CD11b (CR3)-C3 phagocytic pathway directs microglia-mediated synaptic pruning in an activity-dependent manner [46-48]. In addition, complement pathways in the PFC are implicated in the emergence of stressinduced behavioral deficits [14, 49]. These data provide initial evidence that neuronal activity in the medial PFC regulates neuroimmune signaling in the context of chronic stress.

Another important finding in this study is that diazepam limited microglia-mediated neuronal remodeling in lamina I of the medial PFC, which was associated with attenuated dendritic spine deficits in this region following CUS. This supports our prior work in which we found that microglia-mediated neuronal remodeling in lamina I of the medial PFC modulated synaptic deficits after CUS [13]. As noted, microglia are responsive to dynamic changes in neuronal activity $[25,27,50]$, and microglia can contribute to structural remodeling of pre- and post-synaptic elements [12]. Thus, it is plausible that aberrant neuronal activity in response to chronic stress might initiate CSF1 signaling and complement activation in the medial PFC, which provokes microglia-mediated neuronal remodeling. Prior studies also indicate that increased glucocorticoid signaling modulates neurophysiological and neuroimmune responses to stress $[18,51]$. This work is supported by our recent findings that showed blocking stress-induced glucocorticoid signaling prevents microglia-mediated neuronal remodeling in the PFC and associated behavioral consequences [14]. It is also interesting to consider how microglia may shape neuronal structures in other corticolimbic brain regions following chronic stress. Indeed there is evidence that microglia contribute to synaptic deficits in the CA1 region of the HPC in chronic stress [52]. The role of microglia in brain regions that display dendritic hypertrophy following chronic stress (i.e., nucleus accumbens or BLA) remains to be explored. Undoubtedly, brain region-specific cues will direct neuron-microglia interactions in the context of stress [53]. Altogether, these findings indicate that converging molecular and physiological mechanisms contribute to neuronal dysfunction and microglia-mediated neuronal remodeling in chronic stress. Future studies will need to define the neuronand microglia-specific mechanisms that lead to stress-induced synaptic plasticity across corticolimbic brain regions and associated behavioral consequences.

Despite these compelling findings, there are several points that warrant further discussion. One limitation is that these studies were conducted in males only. Chronic stress drives varied neuronal responses in male and females rodents [54-56], and initial sex-dependent differences in microglia likely contribute to divergent microglial responses $[13,57,58]$. Additional experiments will be needed to determine how microglia shape the varied neurobiological effects of stress in males and females. Moreover, other studies have shown that chronic stress alters microglial morphology (IBA1 + area) in the PFC $[13,59,60]$, but in this study we did not observe a significant increase in IBA $1+$ area. These findings are consistent with other reports indicating no change in microglial area $[57,60,61]$. Nonetheless, flow cytometry revealed that CUS increased microglial CD11b levels, and gene expression analyses showed that CUS increased Csf1r and Cd11b levels in sorted PFC microglia. These results indicate that CUS shifted microglia function without causing robust morphological changes in the PFC. It is also important to note that microglia express negligible levels of $\mathrm{GABA}_{\mathrm{A}}$ receptors, suggesting that the effects of diazepam are primarily mediated by actions on neurons $[62,63]$. However, microglia (and endothelial cells) express low levels of the peripheral benzodiazepine receptor (PBR) or translocator protein (TSPO), which can bind diazepam (albeit with low affinity) $[64,65]$. This is pertinent because increased levels of TSPO are observed in several brain regions of individuals suffering from $M D D$, and this is attributed to alterations in microglia function [66]. In this context, it is possible that modulatory effects of diazepam on microglia are mediated, in part, by TSPO. Further studies are needed to determine the role of microglial TSPO in stress responses.

In summary, these studies demonstrate that diazepam modulates chronic stress-induced neuronal activation, and thereby regulates neuron-microglia interactions in the medial PFC. These data provide initial evidence that aberrant neuronal activity in the medial PFC directs microglia-mediated neuronal remodeling and subsequent behavioral consequences. In particular, pharmacological blockade of neuronal activation with diazepam attenuates stress effects on CSF1 and complement signaling, microgliamediated neuronal remodeling, and resultant cognitive and behavioral deficits. Notably, these neuroimmune responses to stress resemble features of an intermediate immune state, termed 
parainflammation, which may be adaptive initially $[67,68]$. Indeed engaging microglia to eliminate hyperactive synaptic connections may help to preserve neuronal integrity. However, with chronic stress microglia-mediated neuronal remodeling may drive pathological synapse loss that contributes to the development of behavioral or cognitive consequences [69]. These findings provide insight into the neurophysiological pathways that provoke alterations in microglia function, and implicate neuronal activitydependent CSF1-CSF1R and complement signaling in synapse loss following chronic stress.

\section{FUNDING AND DISCLOSURE}

This research was supported by a NARSAD Young Investigator Grant and University of Cincinnati Neurobiology Research Center Pilot Grant to ESW. The authors have declared that no conflict of interest exists.

\section{AUTHOR CONTRIBUTIONS}

ESW designed these research studies; JLB, MH, and ESW conducted experiments and acquired data; ESW provided resources and materials; JLB, MH, and ESW analyzed data and wrote the manuscript.

\section{ADDITIONAL INFORMATION}

Supplementary Information accompanies this paper at (https://doi.org/10.1038/ s41386-020-0720-1)

Publisher's note Springer Nature remains neutral with regard to jurisdictional claims in published maps and institutional affiliations.

\section{REFERENCES}

1. Vos T, Allen C, Arora M, Barber RM, Brown A, Carter A, et al. Global, regional, and national incidence, prevalence, and years lived with disability for 310 diseases and injuries, 1990-2015: a systematic analysis for the Global Burden of Disease Study 2015. Lancet. 2016;388:1545-602.

2. Otte C, Gold SM, Penninx BW, Pariante CM, Etkin A, Fava M, et al. Major depressive disorder. Nat Rev Dis Prim. 2016;2:1-20.

3. Wohleb ES, Franklin T, Iwata M, Duman RS. Integrating neuroimmune systems in the neurobiology of depression. Nat Rev Neurosci. 2016;17:497.

4. Christoffel DJ, Golden SA, Russo SJ. Structural and synaptic plasticity in stressrelated disorders. Rev Neurosci. 2011;22:535-49.

5. Kang HJ, Voleti B, Hajszan T, Rajkowska G, Stockmeier CA, Licznerski $P$, et al. Decreased expression of synapse-related genes and loss of synapses in major depressive disorder. Nat Med. 2012;18:1413.

6. Yuen EY, Wei J, Liu W, Zhong P, Li X, Yan Z. Repeated stress causes cognitive impairment by suppressing glutamate receptor expression and function in prefrontal cortex. Neuron. 2012;73:962-77.

7. Ota KT, Liu RJ, Voleti B, Maldonado-Aviles JG, Duric V, Iwata M, et al. REDD1 is essential for stress-induced synaptic loss and depressive behavior. Nat Med. 2014;20:531-5.

8. Tynan RJ, Weidenhofer J, Hinwood M, Cairns MJ, Day TA, Walker FR. A comparative examination of the anti-inflammatory effects of SSRI and SNRI antidepressants on LPS stimulated microglia. Brain Behav Immun. 2012;26:469-79.

9. Wohleb ES, Delpech JC. Dynamic cross-talk between microglia and peripheral monocytes underlies stress-induced neuroinflammation and behavioral consequences. Prog Neuro-Psychopharmacol Biol Psychiatry. 2017;79:40-48.

10. Wake H, Moorhouse AJ, Jinno S, Kohsaka S, Nabekura J. Resting microglia directly monitor the functional state of synapses in vivo and determine the fate of ischemic terminals. J Neurosci. 2009;29:3974-80.

11. Tremblay ME, Lowery RL, Majewska AK. Microglial interactions with synapses are modulated by visual experience. Plos Biol. 2010;8:16.

12. Weinhard L, di Bartolomei G, Bolasco G, Machado P, Schieber NL, Neniskyte U, et al. Microglia remodel synapses by presynaptic trogocytosis and spine head filopodia induction. Nat Commun. 2018;9:1228.

13. Wohleb ES, Terwilliger R, Duman $\mathrm{CH}$, Duman RS. Stress-induced neuronal CSF1 provokes microglia-mediated neuronal remodeling and depressive-like behavior. Biol Psychiatry. 2018;83:38-49.

14. Horchar MJ, Wohleb ES. Glucocorticoid receptor antagonism prevents microgliamediated neuronal remodeling and behavioral despair following chronic unpredictable stress. Brain Behav Immun. 2019. https://doi.org/10.1016/j. bbi.2019.06.030.

15. Arnsten AFT. Stress weakens prefrontal networks: molecular insults to higher cognition. Nat Neurosci. 2015;18:1376-85.

16. Liu R-J, Aghajanian GK. Stress blunts serotonin- and hypocretin-evoked EPSCs in prefrontal cortex: role of corticosterone-mediated apical dendritic atrophy. Proc Natl Acad Sci. 2008;105:359-64.

17. Moghaddam B. Stress preferentially increases extraneuronal levels of excitatory amino acids in the prefrontal cortex: comparison to hippocampus and basal ganglia. J Neurochem. 1993;60:1650-7.

18. Popoli M, Yan Z, McEwen BS, Sanacora G. The stressed synapse: the impact of stress and glucocorticoids on glutamate transmission. Nat Rev Neurosci. 2012;13:22-37.

19. Beck CHM, Fibiger HC. Conditioned fear-induced changes in behavior and in the expression of the immediate early gene c-fos: With and without diazepam pretreatment. J Neurosci. 1995;15:709-20.

20. Delaney AJ, Sah P. GABA receptors inhibited by benzodiazepines mediate fast inhibitory transmission in the central amygdala. J Neurosci. 1999;19:9698-704.

21. Bagley J, Moghaddam B. Temporal dynamics of glutamate efflux in the prefrontal cortex and in the hippocampus following repeated stress: effects of pretreatment with saline or diazepam. Neuroscience. 1997;77:65-73.

22. Finlay JM, Zigmond MJ, Abercrombie ED. Increased dopamine and norepinephrine release in medial prefrontal cortex induced by acute and chronic stress: effects of diazepam. Neuroscience. 1995;64:619-28.

23. Zhao Y, Wang Z, Dai J, Chen L, Huang Y, Zhan Z. Beneficial effects of benzodiazepine diazepam on chronic stress-induced impairment of hippocampal structural plasticity and depression-like behavior in mice. Behav Brain Res. 2012;228:339-50.

24. Shah AA, Treit D. Infusions of midazolam into the medial prefrontal cortex produce anxiolytic effects in the elevated plus-maze and shock-probe burying tests. Brain Res. 2004;996:31-40.

25. Wu Y, Dissing-Olesen L, MacVicar BA, Stevens B. Microglia: dynamic mediators of synapse development and plasticity. Trends Immunol. 2015;36:605-13.

26. Eyo UB, Gu N, De S, Dong HL, Richardson JR, Wu LJ. Modulation of microglial process convergence toward neuronal dendrites by extracellular calcium. J Neurosci. 2015;35:2417-22.

27. Dissing-Olesen L, LeDue JM, Rungta RL, Hefendehl JK, Choi HB, MacVicar BA. Activation of neuronal NMDA receptors triggers transient ATP-mediated microglial process outgrowth. J Neurosci. 2014;34:10511.

28. Luo J, Elwood F, Britschgi M, Villeda S, Zhang H, Ding Z, et al. Colony-stimulating factor 1 receptor (CSF1R) signaling in injured neurons facilitates protection and survival. J Exp Med. 2013;210:157-72.

29. laccarino HF, Singer AC, Martorell AJ, Rudenko A, Gao F, Gillingham TZ, et al. Gamma frequency entrainment attenuates amyloid load and modifies microglia. Nature. 2016;540:230-5.

30. Liu RJ, Duman C, Kato T, Hare B, Lopresto D, Bang E, et al. GLYX-13 produces rapid antidepressant responses with key synaptic and behavioral effects distinct from ketamine. Neuropsychopharmacology. 2017;42:1231-42.

31. O'Connor KA, Mahoney E, Ramirez-Zamora A, Molho ES, Pilitsis JG, Shin DS. Effect of diazepam and yohimbine on neuronal activity in sham and hemiparkinsonian rats. Neuroscience. 2017;351:71-83.

32. Conrad CD, Ortiz JB, Judd JM. Chronic stress and hippocampal dendritic complexity: methodological and functional considerations. Physiol Behav. 2017;178:66-81.

33. Vyas A, Mitra R, Rao BSS, Chattarji S. Chronic stress induces contrasting patterns of dendritic remodeling in hippocampal and amygdaloid neurons. J Neurosci. 2002;22:6810-8.

34. Vyas A, Chattarji S. Modulation of different states of anxiety-like behavior by chronic stress. Behav Neurosci. 2004;118:1450-4.

35. Shors TJ, Chua C, Falduto J. Sex differences and opposite effects of stress on dendritic spine density in the male versus female hippocampus. J Neurosci. 2001;21:6292-7.

36. Hercher C, Turecki G, Mechawar N. Through the looking glass: examining neuroanatomical evidence for cellular alterations in major depression. J Psychiatr Res. 2009;43:947-61.

37. Holmes SE, Scheinost D, Finnema SJ, Naganawa M, Davis MT, DellaGioia N, et al. Lower synaptic density is associated with depression severity and network alterations. Nat Commun. 2019;10:1529.

38. Radley JJ, Rocher AB, Miller M, Janssen WGM, Liston C, Hof PR, et al. Repeated stress induces dendritic spine loss in the rat medial prefrontal cortex. Cereb Cortex. 2006;16:313-20.

39. Cook SC, Wellman CL. Chronic stress alters dendritic morphology in rat medial prefrontal cortex. J Neurobiol. 2004;60:236-48.

40. Dwyer JM, Maldonado-Aviles JG, Lepack AE, DiLeone RJ, Duman RS. Ribosomal protein S6 kinase 1 signaling in prefrontal cortex controls depressive behavior. Proc Natl Acad Sci USA. 2015;112:6188-93. 
1776

41. Bollinger JL, Wohleb ES. The formative role of microglia in stress-induced synaptic deficits and associated behavioral consequences. Neurosci Lett. 2019;711:134369.

42. Lee HK, Dunwiddie TV, Hoffer BJ. Interaction of diazepam with synaptic transmission in the in vitro rat hippocampus. Naunyn Schmiedebergs Arch Pharm. 1979;309:131-6.

43. Petty F, Jordan S, Kramer GL, Zukas PK, Wu J. Benzodiazepine prevention of swim stress-induced sensitization of cortical biogenic amines: an in vivo microdialysis study. Neurochem Res. 1997;22:1101-4.

44. De I, Nikodemova M, Steffen MD, Sokn E, Maklakova VI, Watters JJ, et al. CSF1 overexpression has pleiotropic effects on microglia in vivo. Glia. 2014;62:1955-67.

45. Elmore MR, Najafi AR, Koike MA, Dagher NN, Spangenberg EE, Rice RA, et al. Colony-stimulating factor 1 receptor signaling is necessary for microglia viability, unmasking a microglia progenitor cell in the adult brain. Neuron. 2014;82:380-97.

46. Stevens B, Allen NJ, Vazquez LE, Howell GR, Christopherson KS, Nouri N, et al. The classical complement cascade mediates CNS synapse elimination. Cell. 2007;131:1164-78.

47. Schafer DP, Lehrman EK, Kautzman AG, Koyama R, Mardinly AR, Yamasaki R, et al. Microglia sculpt postnatal neural circuits in an activity and complementdependent manner. Neuron. 2012;74:691-705.

48. Hong S, Beja-Glasser VF, Nfonoyim BM, Frouin A, Li S, Ramakrishnan S, et al. Complement and microglia mediate early synapse loss in Alzheimer mouse models. Science. 2016;352:712-6.

49. Crider A, Feng T, Pandya CD, Davis T, Nair A, Ahmed AO, et al. Complement component 3a receptor deficiency attenuates chronic stress-induced monocyte infiltration and depressive-like behavior. Brain Behav Immun. 2018;70:246-56.

50. Eyo UB, Peng J, Swiatkowski P, Mukherjee A, Bispo A, Wu L-JJ. Neuronal hyperactivity recruits microglial processes via neuronal NMDA receptors and microglial P2Y12 receptors after status epilepticus. J Neurosci. 2014;34:10528-40.

51. Frank MG, Hershman SA, Weber MD, Watkins LR, Maier SF. Chronic exposure to exogenous glucocorticoids primes microglia to pro-inflammatory stimuli and induces NLRP3 mRNA in the hippocampus. Psychoneuroendocrinology. 2014:40:191-200.

52. Milior G, Lecours C, Samson L, Bisht K, Poggini S, Pagani F, et al. Fractalkine receptor deficiency impairs microglial and neuronal responsiveness to chronic stress. Brain Behav Immun. 2016;55:114-25.

53. De Biase LM, Schuebel KE, Fusfeld ZH, Jair K, Hawes IA, Cimbro R, et al. Local cues establish and maintain region-specific phenotypes of basal ganglia microglia. Neuron. 2017;95:341-356.e6.

54. Garrett JE, Wellman CL. Chronic stress effects on dendritic morphology in medial prefrontal cortex: sex differences and estrogen dependence. Neuroscience. 2009;162:195-207.
55. Shansky RM, Hamo C, Hof PR, Lou W, McEwen BS, Morrison JH. Estrogen promotes stress sensitivity in a prefrontal cortex-amygdala pathway. Cereb Cortex. 2010;20:2560-7.

56. Moench KM, Wellman CL. Differential dendritic remodeling in prelimbic cortex of male and female rats during recovery from chronic stress. Neuroscience. 2017:357:145-59.

57. Bollinger JL, Salinas I, Fender E, Sengelaub DR, Wellman CL. Gonadal hormones differentially regulate sex-specific stress effects on glia in the medial prefrontal cortex. J Neuroendocrinol. 2019;31:e12762:1-19.

58. Fonken LK, Frank MG, Gaudet AD, D'Angelo HM, Daut RA, Hampson EC, et al. Neuroinflammatory priming to stress is differentially regulated in male and female rats. Brain Behav Immun. 2018;70:257-67.

59. Hinwood M, Morandini J, Day TA, Walker FR. Evidence that microglia mediate the neurobiological effects of chronic psychological stress on the medial prefrontal cortex. Cereb Cortex. 2012;22:1442-54.

60. Kopp BL, Wick D, Herman JP. Differential effects of homotypic vs. heterotypic chronic stress regimens on microglial activation in the prefrontal cortex. Physiol Behav. 2013;122:246-52.

61. Bollinger JL, Burns CMB, Wellman CL. Differential effects of stress on microglial cell activation in male and female medial prefrontal cortex. Brain Behav Immun. 2016;52:88-97.

62. Tasic B, Menon V, Nguyen TN, Kim TK, Jarsky T, Yao Z, et al. Adult mouse cortical cell taxonomy revealed by single cell transcriptomics. Nat Neurosci. 2016;19:335-46.

63. Zhang Y, Chen K, Sloan SA, Bennett ML, Scholze AR, O'Keeffe S, et al. An RNAsequencing transcriptome and splicing database of glia, neurons, and vascular cells of the cerebral cortex. J Neurosci. 2014;34:11929-47.

64. Notter T, Coughlin JM, Sawa A, Meyer U. Reconceptualization of translocator protein as a biomarker of neuroinflammation in psychiatry. Mol Psychiatry. 2018;23:36-47.

65. Betlazar C, Harrison-Brown M, Middleton RJ, Banati R, Liu GJ. Cellular sources and regional variations in the expression of the neuroinflammatory marker translocator protein (TSPO) in the normal brain. Int J Mol Sci. 2018;19:1-18.

66. Setiawan E, Wilson AA, Mizrahi R, Rusjan PM, Miler L, Rajkowska G, et al. Role of translocator protein density, a marker of neuroinflammation, in the brain during major depressive episodes. JAMA Psychiatry. 2015;72:268-75.

67. Medzhitov R. Origin and physiological roles of inflammation. Nature. 2008;454:428-35.

68. Kotas ME, Medzhitov R. Homeostasis, inflammation, and disease susceptibility. Cell. 2015;160:816-27.

69. Wohleb ES. Neuron-microglia interactions in mental health disorders: 'For better, and for worse'. Front Immunol. 2016;7:1-49. 